\title{
Análise do Controle Motor com Interações baseadas em Dispositivos Convencionais e Realidade Aumentada ${ }^{1}$
}

\author{
Marcelo da S. Hounsell ${ }^{2}$ \\ Débora C. Xavier \\ Tânia B. Crocetta ${ }^{3}$ \\ Alexandro Andrade ${ }^{3}$
}

\begin{abstract}
Resumo: Este trabalho apresenta um software para auxílio ao diagnóstico dos problemas do controle motor fino em humanos, denominado MOSKA ( $\underline{\text { MOtor }}$

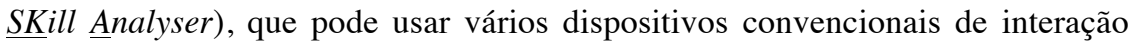
(como mouse e mesa digitalizadora - tablet) e Realidade Aumentada (RA) (baseada em dispositivos de baixo custo como webcam). Duas técnicas de interação, com RA e sem RA, foram implementadas com o objetivo de capturar métricas, já validadas em pesquisas anteriores, relativas ao tempo de duração e precisão de desenhos produzidos pelos participantes. O desenvolvimento levou a um sistema que dispõe de métricas novas e mais significativas além de um Banco de Dados que permitiram efetuar uma comparação entre as técnicas de interação para este tipo de diagnóstico. As implementações foram feitas em Java e, para RA, foi usada a biblioteca jARToolKit por ser popular, livre e de código
\end{abstract}

1 Este trabalho foi parcialmente publicado no SCA 2009 e foi selecionado como um dos três melhores artigos do evento.

${ }^{2}$ Laboratory for Research on Visual Applications, LARVA - DCC, UDESC, Campus Universitário Avelino Marcante S/N, Bom Retiro, 89223-100, Joinville, SC, Brasil. \{deboracxavier@ gmail.com, marcelo@joinville.udesc.br\}

${ }^{3}$ Laboratório de Psicologia do Esporte e do Exercício, LAPE - UDESC, Rua Pascoal Simone 358, Bairro Coqueiros, 88080-350, Florianópolis, SC, Brasil. \{d4tbc,d2aa\}@udesc.br 
aberto mas que implica no uso de marcadores, o que levou a identificar algumas limitações deste tipo de RA. Apesar de certo entusiasmo por ser uma tecnologia mais moderna, descobriu-se que certas técnicas específicas de RA não são apropriadas para aplicações que necessitem de precisão e coordenação visualmotora, como a análise do controle motor fino.

\begin{abstract}
This work presents a software, named MOSKA (MOtor $\underline{\text { SKill }}$ Analyser), to help diagnose human's fine motor control problems that can use a variety of conventional interaction devices such as mouse and tablet, and Augmented Reality (AR), based on low cost devices such as webcams. Two interaction techniques have been implemented, with and without $\mathrm{AR}$, aiming at gathering measurements which have already been validated, concerning time duration and precision of patient's drawings. The development produced a system that provides new and more meaningful measurements as well as a Data Base that allowed a comparison between interaction techniques. Implementations were carried out using Java and the jARToolKit library which were used due to their popularity, open and free codes. Although there exist some hype because it represents a modern approach, it was found that some AR setup are not suitable for those applications that require precision and visualmotor coordination such as for fine motor control analysis.
\end{abstract}

\title{
1 Introdução
}

Várias são as causas para os distúrbios do controle motor. Desde degenerações neurológicas, Acidentes Vasculares Cerebrais (AVCs) até lesões por esforços repetitivos e cotidianos passando pelo estresse oriundo de uma competição, todos podem ter reflexos no controle motor. Estes são apenas alguns exemplos que acabam por requerer intervenções médicas no sentido de reabilitar o paciente. Neste processo de reabilitação destacam-se duas fases: a fase diagnóstica (responsável por levantar dados e interpretá-los) e; a fase terapêutica (responsável por recuperar as funções perdidas ou comprometidas). 
O uso de técnicas computacionais de interação, baseadas em rastreamento de posição, reconhecimento de gestos e posturas, dentre outras, podem criar um ambiente mais descontraído, motivador e intuitivo pois facilitam o reconhecimento dos movimentos do paciente como entrada de dados para o sistema e então, a partir destas, inferir informações pertinentes ao processo fisioterápico.

A informática pode auxiliar na reabilitação do controle motor em, pelo menos, dois aspectos: no armazenamento e recuperação de dados (do participante, da patologia, da terapia, do terapeuta, etc.) e; na captura e tratamento de medidas específicas (no processo de facilitação da obtenção, no tratamento, conversão, interpretação, etc.). Os primeiros aspectos se beneficiam da já madura tecnologia de Banco de Dados ao passo que os últimos podem se beneficiar de uma ampla miríade de novas tecnologias que surgiram recentemente, como Realidade Virtual (RV), Visão Computacional, Inteligência Artificial, dentre outras.

A RV é definida como uma mídia composta de simulações computacionais interativas que percebe as posições e ações do participante e substitui ou aumenta o retorno (feedback) para um ou mais sentidos produzindo a sensação de estar mentalmente imerso ou presente no mundo virtual [Sherman \& Craig, 2003: 13]. Esta técnica simula o mundo real utilizando conceitos de computação gráfica, permitindo assim, uma interação intuitiva utilizando dispositivos como capacetes de visualização (HMDs - Head Mounted Displays), luvas de dados (Data Gloves) ou o próprio corpo do usuário.

Dentro deste contexto, existe também a Realidade Aumentada (RA) que consiste na utilização do mundo real como ambiente, sendo que neste é possível visualizar e interagir com objetos virtuais, dando a ilusão de que o mundo virtual e real coexistem. A RA consiste num tipo de RV na qual os estímulos sintéticos (objetos virtuais, por exemplo) são sobrepostos a objetos no mundo real [Sherman \& Craig, 2003: 18].

Portanto, pode-se esperar que a RA leve a uma interação intuitiva pelo fato de que a interface é o ambiente real e objetos virtuais podem ser inseridos neste ambiente para simular alguma situação significativa. Deste modo, técnicas de interação intuitivas poderiam se basear na RA para auxiliar na análise do controle motor.

A RV e RA disponibilizam técnicas de interação que permitem simular situações do cotidiano, assim como oportunidades de envolvimento em comportamentos e desafios, porém realizados com segurança. Além disso, a RV e a RA fornecem meios de diagnosticar e tratar patologias relacionadas ao controle motor dentro de um contexto funcional focado no tratamento (interessante e motivador para o participante) possibilitando ainda a classificação e documentação dos mesmos [Sveistrup, 2004].

O Controle Motor (CM) pode ser definido como a capacidade de coordenar movimentos gerados pela integração entre um comando cerebral e as unidades motoras dos músculos e articulações [Sanches, 2007]. Assim, o CM é um conjunto de ações que se iniciam na percepção do estímulo e envolve o sistema nervoso como um todo. O CM pode ser classificado em CM grosso ou fino [Bueno, 1998]: 
- O CM grosso envolve os movimentos amplos (caminhar e correr, por exemplo) que utilizam a contração de grandes músculos corporais;

- O CM fino é a capacidade de controlar os pequenos músculos para movimentos delicados e específicos (escrever, recortar, colar e pegar, por exemplo) e envolve a coordenação visual-motora (capacidade de coordenar movimentos em relação a um alvo visual), visual-manual (coordenação entre visão e tato) e músculo-facial (movimentos finos da face).

Este trabalho apresentará o software MOSKA (MOtor SKill Analyser) que representa a implementação de soluções da área de informática (notadamente Banco de Dados) e computação (incluindo RA) no desenvolvimento de um sistema para auxílio ao diagnóstico do controle motor fino. Técnicas de interação, em especial o uso da RA com marcadores e equipamentos de baixo custo com bibliotecas livres e gratuitas, serão detalhadas e discutidas.

\section{Trabalhos Relacionados}

O trabalho proposto por Viau e colegas (2004) objetivou validar a RV como uma ferramenta de estudo do movimento da extensão do braço e da preensão (CM fino). Para isto, os autores compararam a cinemática do movimento em tarefas idênticas em um ambiente físico e virtual (este último usando luva de dados com retorno tátil e visualização em monitor 2D convencional sem recurso de estereoscopia). A tarefa consistia em pegar uma bola (real ou virtual), levá-la a uma determinada posição e largá-la.

Os autores concluíram que o ambiente virtual proporcionou uma diminuição na extensão do pulso e um aumento na extensão do cotovelo, quando comparado ao ambiente físico, devido à ausência das percepções da profundidade e do retorno tátil ao liberar a bola no ambiente virtual. Assim, os autores sustentam que o ambiente físico apresenta melhores resultados que o ambiente virtual, porém o trabalho demonstrou que há semelhanças importantes e vantagens em alguns aspectos e sugerem que um ambiente virtual 3D imersivo poderia ter resultados melhores que o ambiente físico, pois este solucionaria os problemas do ambiente virtual usado (com visualização em tela 2D não estereoscópica).

Luo e colegas (2005) desenvolveram um ambiente de treinamento para extensão de dedos (CM fino) que utiliza RA e dispositivos de assistência para movimentar os dedos. Esta aplicação usufruiu de dispositivos para RA, que incluem um HMD com visor LCD semitransparente estéreo e com rastreador de posição, e ainda, de dispositivos ortopédicos atuadores, baseados em cabos ou bolhas de ar. A tarefa era pegar e largar objetos virtuais e foram medidos velocidade e deslocamento máximo. Os resultados dos experimentos mostraram que o treinamento apresenta uma "tendência encorajadora" no auxílio da melhora da capacidade de extensão dos dedos da mão afetada. Isso demonstra a possibilidade real do uso da tecnologia de RA com dispositivos assistivos para a reabilitação do CM fino. 
Eder e colegas (2005) desenvolveram o Drawing Test (Teste do Desenho) que utiliza uma mesa digitalizadora (tablet) com um mouse para avaliar movimentos do ombro e cotovelo juntos durante a execução de tarefas. A tarefa consistia no desenho sobre uma mesa digitalizadora em que o indivíduo deveria realizar os movimentos dos cantos inferiores aos cantos superiores de um quadrado seguindo as arestas laterais (de A a B, e de D a C na Figura 1). Segundo os autores, o Drawing Test foi validado para a medição do CM fino em indivíduos hemiplégicos pós-AVC e os resultados mostraram "forte correlação" entre o Drawing Test (e as métricas do erro final de posição e do desvio padrão da aresta de referência) e a Escala de Ashworth, que é uma medição clínica de espasticidade (rigidez muscular).

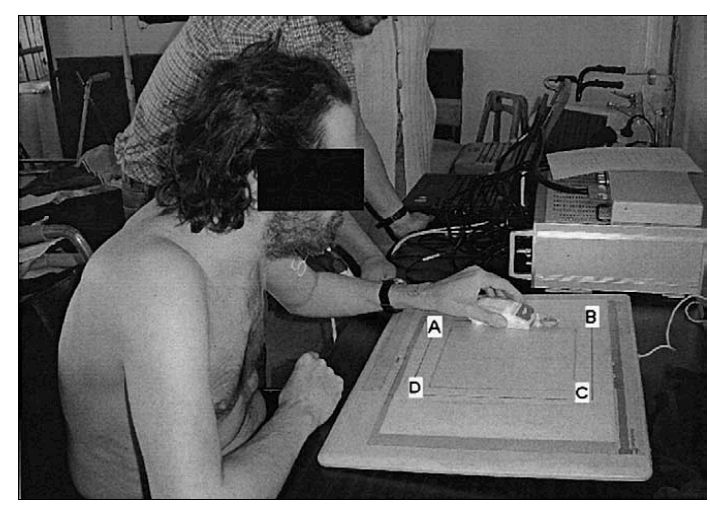

Figura 1. O Drawing Test [Eder et al, 2005]

Subramanian e colegas (2007) apresentaram um ambiente físico e outro de RV (com HMD estéreo, rastreadores de posição e luva de dados) que induz a prática de movimentos necessários para a recuperação motora reproduzindo um ambiente assemelhado ao de um elevador e seus botões (CM fino). Seis botões foram dispostos à frente do paciente e um deles era indicado para que o paciente o tocasse. Foram medidas as posições dos dedos, braço e tronco, suas velocidades máximas e o erro final de posição. Os autores concluíram que o movimento tende a ser mais lento, menos preciso e mais curvado no ambiente virtual que no real. Entretanto, todos os participantes relataram que o ambiente virtual foi mais interessante e motivante que o físico e que isso os encorajou a realizar mais exercícios.

Sanches (2007) avaliou a relação entre CM, dor e depressão em mulheres com fibromialgia. Foi utilizado o "Software de Avaliação da Aprendizagem e Controle Motor" (ACM) [Andrade et. al., 2003] com uma mesa digitalizadora e uma caneta para a realização dos testes e foi calculado o tempo de duração da tarefa e a quantidade de erro ao final da mesma. A tarefa consistia em desenhar certas retas sobre a mesa digitalizadora. Observou-se que o valor médio de erro nas avaliações das participantes foi proporcional ao grau de dificuldade das tarefas. Com este estudo, pôde-se afirmar que tarefas simples de desenho foram validadas para mulheres com fibromialgia como forma de avaliar o CM fino. 
A Tabela 1 mostra uma comparação dos sistemas apresentados. Estão ressaltadas as características dos dispositivos usados, o tipo de ambiente e o tipo de uso de cada pesquisa. Quanto aos dispositivos, observa-se o uso de uma ampla variedade deles, desde HMDs estéreos (e caros), dispositivos e interação convencional (e baratos, como mouse e tablet), dispositivos de retorno de força (hápticos) e captura de configurações da mão (luvas). Os tipos de ambiente encontrados incluem desde ambientes físicos (não criados artificialmente em computador) até ambientes de RV e RA.

As pesquisas apresentadas trataram do CM fino com foco mas que variaram de ambientes voltados para a "avaliação", ambientes voltados para o processo de "reabilitação" ou ainda, ambientes cujo objetivo era "comparar" situações entre o mundo virtual e real/físico.

Os trabalhos apresentados demonstram que a RV e a RA têm sido validadas como tecnologias facilitadoras do processo terapêutico (tanto na análise quanto no trato) de problemas de CM. Entretanto, nenhum dos trabalhos identificados abordou o uso da RA de baixo custo (com webcam, mouse, tablet e bibliotecas de software abertas, gratuitas e de uso geral para RA) em aplicações de diagnóstico (avaliação) do CM fino e portanto, este trabalho visa explorar estas possibilidades. Na última coluna da Tabela 1, tem-se indicadas as características do sistema proposto por esta pesquisa.

Tabela 1. Comparação dos Sistemas (Adaptado de [Xavier, 2007]).

\begin{tabular}{|c|c|c|c|c|c|c|c|}
\hline \multicolumn{2}{|r|}{ Elemento } & Viau & Luo & Eder & Subramanian & Sanches & MOSKA \\
\hline \multirow{5}{*}{ Dispositivos } & HMD & & $\mathrm{x}$ & & $\mathrm{x}$ & & \\
\hline & Câmera de Vídeo & & & & & & $\mathrm{x}$ \\
\hline & Mouse/Tablet & & & $\mathrm{x}$ & & & $\mathrm{x}$ \\
\hline & Háptico & $\mathrm{x}$ & $\mathrm{x}$ & & & & \\
\hline & Luva & $\mathrm{x}$ & $\mathrm{x}$ & & $\mathrm{x}$ & & \\
\hline \multirow{3}{*}{ Ambiente } & Real/Físico & & & $\mathrm{x}$ & & $\mathrm{x}$ & \\
\hline & RV & $\mathrm{x}$ & & & $\mathrm{x}$ & & \\
\hline & RA & & $\mathrm{x}$ & & & & $x$ \\
\hline \multirow{3}{*}{ Uso } & Avaliação & & & $x$ & & $x$ & $x$ \\
\hline & Reabilitação & & $\mathrm{x}$ & & $x$ & & \\
\hline & Comparação Real e Virtual & $\mathrm{x}$ & & & $\mathrm{x}$ & & \\
\hline
\end{tabular}




\section{Melhorias na Análise do Controle Motor}

O software de "Avaliação da Aprendizagem e Controle Motor" (ACM) [Andrade et. al., 2003] avalia as habilidades motoras finas, discretas e fechadas de membros superiores. $\mathrm{O}$ objetivo do sistema é o deslocamento do cursor pelo participante, de um ponto inicial a um ponto final de uma linha reta pré-determinada no sistema. Sanches (2007), em seu estudo piloto, usou o ACM e definiu as tarefas, seu nível de dificuldade e a quantidade de tentativas requeridas a cada uma. A autora demonstrou que tarefas "aparentemente" simples (desenho de retas) podem ser úteis para um processo diagnóstico sério e rigoroso.
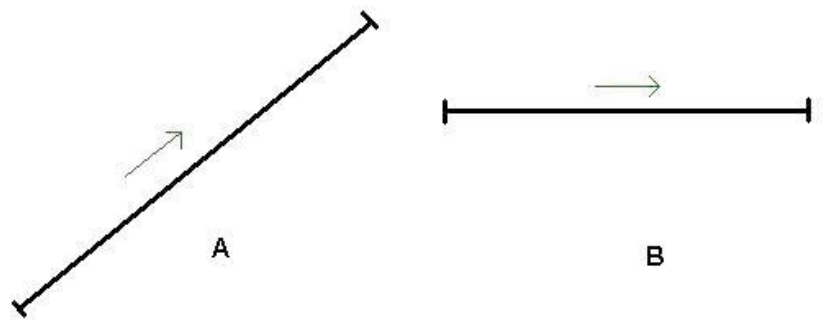

Figura 2. Tarefas de avaliação do controle motor [Sanches, 2007]

A Figura 2 mostra o movimento que deve ser executado em cada tarefa: (A) movimento inclinado ascendente à direita; (B) movimento horizontal à direita, e; (C) movimento sagital póstero-anterior. Estas tarefas serão utilizadas neste estudo como sendo as tarefas básicas para a avaliação do CM fino.

Foi tomado o software ACM como referência para este trabalho por ter tarefas e métricas já validadas ([Sanches, 2007] e [Gonçalves, 2008]), por ter sido proposto por pesquisadores brasileiros da área da saúde ([Andrade et. al., 2003], [Jardim, 2008]) e por estes pesquisadores terem disponibilizado o código para análises e contribuído para esta pesquisa. Foi constatado que as técnicas de interação do ACM (com mouse e tablet) poderiam ser ampliadas com o uso de novas tecnologias, em especial a RA. A técnica de RA foi escolhida, pois sua interação contempla o uso de cenas e objetos físicos reais, concomitantemente a objetos virtuais e, se forem usados recursos de marcadores, isso pode ser alcançado com baixo custo. Em contato com os pesquisadores envolvidos no projeto do ACM ficou claro que o uso de RA representava uma promessa de melhoria significativa na interação, tornando o sistema intuitivo e motivador (pelo uso de objetos virtuais 3D e um recurso tecnológico atual).

Outras melhorias incorporadas incluíram o armazenamento e recuperação de dados sobre os participantes, testes, a possibilidade de definir novas tarefas (novos segmentos de retas em posições específicas), consulta e geração de gráficos e também o cálculo de várias outras métricas temporais e espaciais. Estas melhorias funcionais juntas à reformulação do visual e navegação da interface fez gerar o software MOSKA, que será detalhado adiante. 


\section{MOSKA (MOtor SKill $\underline{\text { Analyser })}$}

A Figura 3 mostra as principais telas do MOSKA que são: a tela inicial (a); a tela de cadastro de tarefas (b); de cadastro de uma nova tarefa; (c) a de relatórios de vários testes e; (d) a tela de realização de um teste.

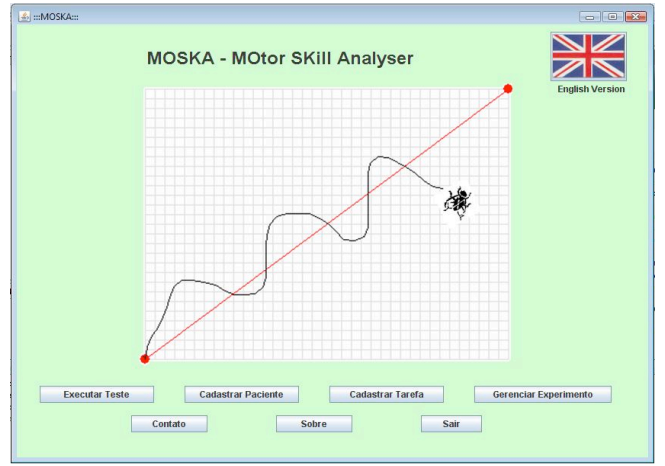

(a)

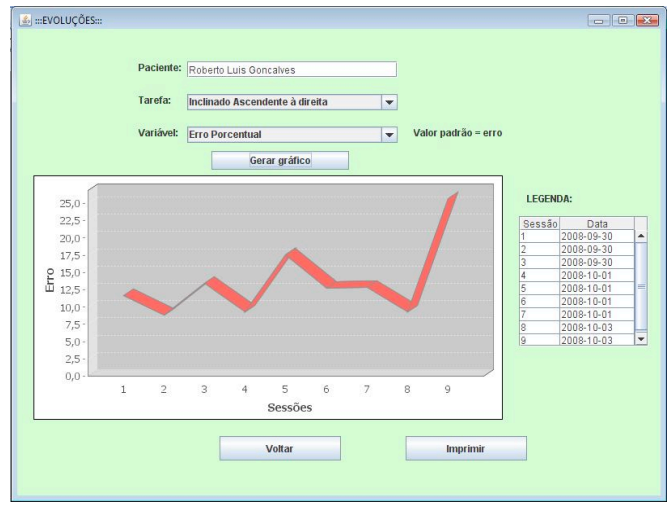

(c)

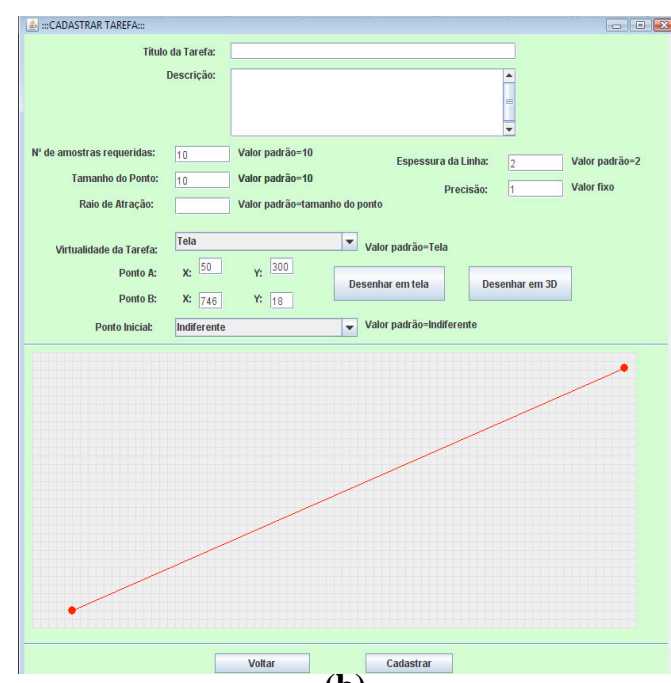

(b)

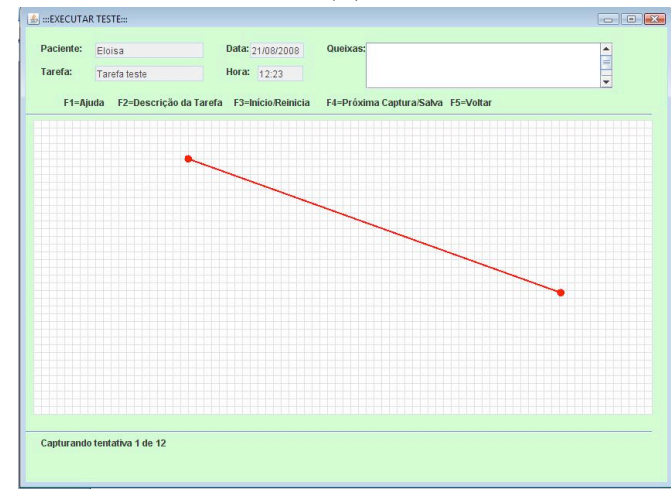

(d)

Figura 3. Principais Telas do MOSKA [Heidemann, 2008]

O MOSKA foi implementado em Java para seguir a mesma tecnologia utilizada pelo ACM utilizou esta mesma tecnologia. Algumas melhorias incorporadas incluem:

- Realização dos testes sempre com a precisão máxima; 
- Gravação dos dados e resultados dos testes em um Banco de Dados, e;

- Possibilidade de cadastro de participantes, testes e tarefas.

A captura de dados motores no MOSKA dispõe de três versões diferentes de interação: uma versão tablet/mouse; uma versão RA 2D e; uma versão RA 3D. As versões RA 2D e 3D utilizam Realidade Aumentada, sendo que a diferença se dá pela dimensionalidade da tarefa/interação, ou seja, na versão RA 2D a tarefa é exibida ao participante coincidente com o plano da superfície de uma mesa e, na versão RA 3D, a tarefa se apresenta no espaço 3D [Xavier, 2008].

\subsection{A Versão Tablet/Mouse}

A versão tablet/mouse permite usar um dispositivo ou o outro numa mesma interface. Com relação ao mouse, deve-se escolher o ótico sem fio para se obter "maior precisão, fidedignidade e conforto, [...] e por não possuir componentes mecânicos como os presentes no mouse eletrônico, evitando problemas de acúmulo de poeira nos roletes internos [Andrade e Haupenthal, 2001] por ser desprovido destes. O fato de não ter fio acarreta em maior validade ecológica, pois o movimento não sofre a interferência do cabo que conecta o mouse ao microcomputador" [Jardim, 2008].

Entretanto, analisando as características dos dispositivos mouse e tablet, deve-se sempre preferir o tablet, pois além de possibilitar "a adaptação de participantes ao uso da caneta, enquanto que, o uso do mouse poderia beneficiar as mulheres [i. e.: participantes] mais adaptadas à utilização de computadores" [Sanches, 2007: 47], deve-se considerar também que o mouse é um dispositivo de posicionamento relativo ao passo que o tablet é de posicionamento absoluto, o que garante maior fidedignidade do desenho, em todas as situações. A Figura 3.b mostra a interface para definição de uma tarefa (a ser executada durante um teste de CM) para a versão mouse/tablet e a Figura 3.d mostra a tela de execução deste teste.

As mensurações que eram tomadas antes foram copiados integralmente (evitando quaisquer discrepâncias com o que já se tinha), inclusive usando o mesmo código, entretanto as seguintes métricas foram incluídas:

- Cálculo do erro como uma percentagem em relação ao caminho de referência. Este cálculo retorna um valor percentual indicando a relação entre o desenho feito pelo participante e o número de pixels contido no desenho de referencia da tarefa (uma reta). Esta medida é portanto, independente da resolução do monitor sendo usado;

- Cálculo a velocidade média e máxima. A velocidade média é calculada pelo tempo total e o comprimento total do desenho gerado pelo participante ao passo que a máxima é calculada com base no espaço a intervalos fixos de tempo; 
- Cálculo do erro entre a linha de referência e a linha desenhada pelo participante através da área entre essas linhas. $\mathrm{O}$ erro em área, na verdade, calcula a média dos erros acumulados ao longo do desenho (ocorrendo tanto no plano - no caso das versões mouse/tablet e RA 2D, quanto no espaço 3D como na versão RA 3D). Este valor portanto, equivale ao desvio padrão do desenho que então, complementa estatisticamente a medida do erro percentual.

\subsection{A Versão RA 2D}

Esta versão usa a RA para realizar a análise do CM fino de forma semelhante à versão mouseltablet, porém a mesa digitalizadora e a caneta passam a ser objetos virtuais. Esta versão utilizou a tecnologia e conceitos do ARToolKit [Hitlab, 2008] para seu desenvolvimento.

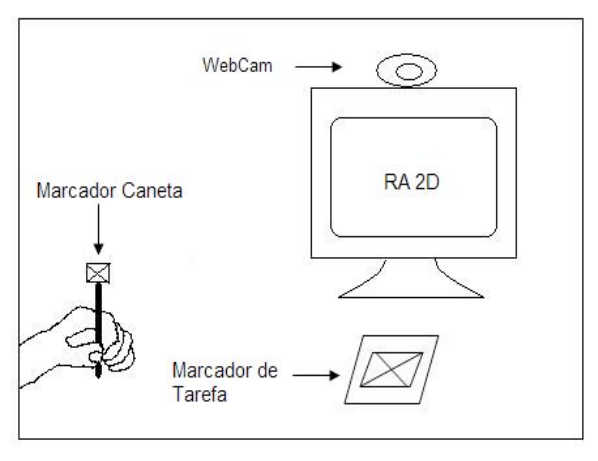

(a)

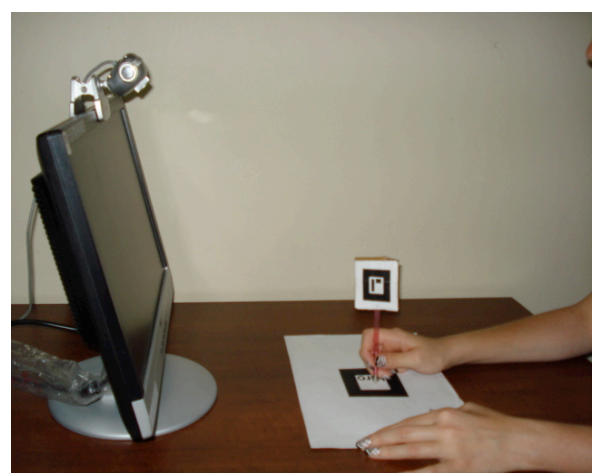

(b)

Figura 4. Ambiente de teste das versões com RA [Xavier, 2008]

O biblioteca ARToolKit tem sido considerada como a responsável pela popularização da tecnologia da RA por ser gratuita, de código aberto, fácil de usar, de configurar e utiliza webcam convencionais (de baixo custo e facilmente disponíveis). O ARToolKit, por sua vez, adota a tecnologia de visão computacional que requer o uso de marcadores (templates padrões impressos em papel) que, quando inseridos na cena e visualizados pela câmera, são identificados e produzem a inserção de um objeto virtual na cena gráfica mostrada no monitor, coerentes com a posição do marcador na cena. No caso, um marcador foi usado para representar uma mesa digitalizadora (tablet) virtual com a identificação da tarefa inserida nesta. Um outro marcador fixado em uma haste real representa uma caneta virtual, e esta se movimenta de acordo com os movimentos do participante (capturados quando dentro do campo de visão da webcam).

Para realizar o teste foi composto um ambiente, conforme esquematizado na Figura 4.a, que serve tanto para a versão RA 2D quanto RA 3D. Na Figura 4.b, tem-se a visão lateral do 
teste real onde o participante fica de frente a um monitor de computador equipado onde a cena é mostrada e a webcam é disposta de forma a visualizar os dois marcadores utilizados e na frente do monitor.

\subsection{A Versão RA 3D}

Em termos de implementação da interação no software não há diferença entre a versão RA 2D e RA 3D.

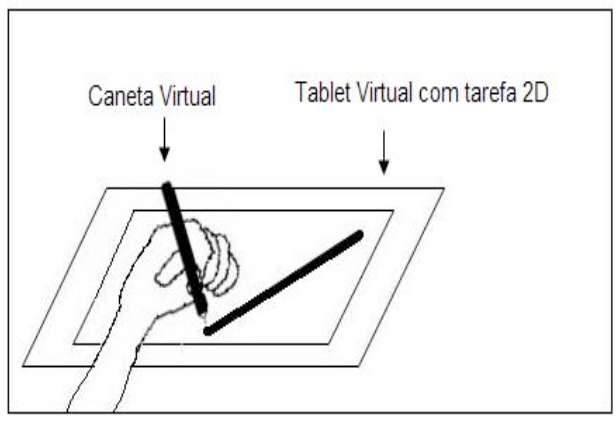

(a)

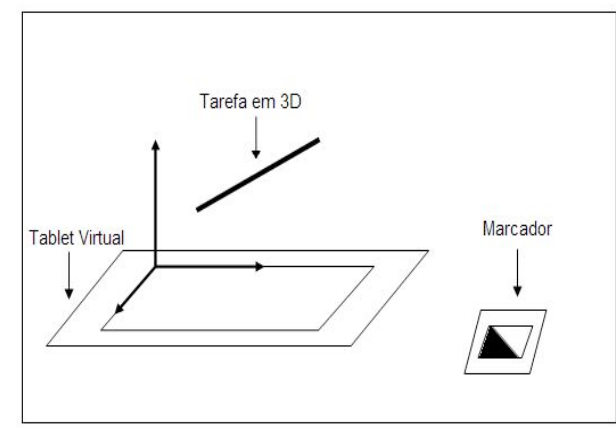

(b)

Figura 5. Diferença entre as versões RA 2D (a) e RA 3D (b) [Xavier, 2008]

A diferença está no fato de que na versão 2D a tarefa é executada sobre a mesa digitalizadora, sendo que na versão RA 3D a tarefa é executada no espaço 3D. Isto significa que muda somente o modelo tridimensional que representa a mesa digitalizadora e que contém o desenho da tarefa (reta) que deixa de ser também no plano da mesa (na versão $2 \mathrm{D}$, como mostra a Figura 5.a) para ser disposto no espaço (na versão 3D, como mostra a Figura 5.b). Entretanto, a disposição física dos elementos no ambiente real de realização do teste é o mesmo utilizado na versão RA 2D (ver Figura 4.b).

Os movimentos do marcador da caneta capturados pela webcam são identificados e substituídos pelo sistema por uma caneta virtual gerando uma linha virtual representativa do movimento. Na versão RA 3D, diferente das demais, as medidas do $\mathrm{CM}$ passam a ser calculadas no espaço euclidiano 3D e não mais num plano de referência.

\section{Implementação}

Utilizou-se a biblioteca de software jARToolKit [SourceForge.net, 2008] para a implementação das versões RA, pois esta é a versão em Java do ARToolKit. A escolha desta biblioteca foi devida a integração de todas as versões em uma única plataforma, gerando um único sistema com o software legado. 


\subsection{Objetos Virtuais}

Tanto o jARToolKit quanto o ARToolKit não possuem métodos nativos para carregar objetos virtuais.

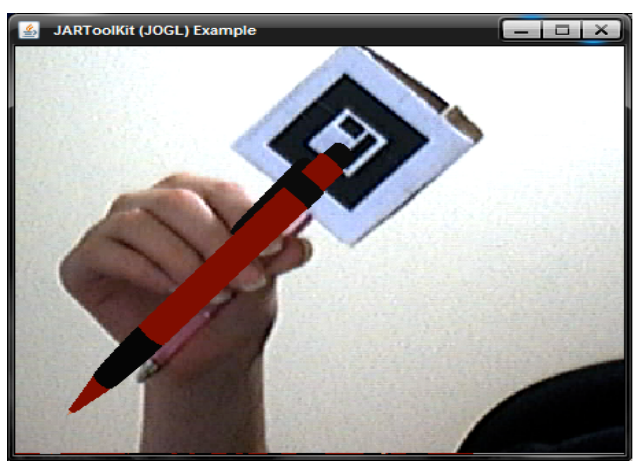

(a)

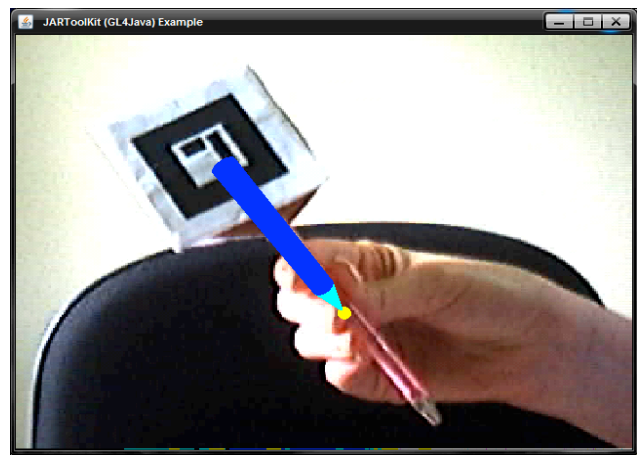

(c)

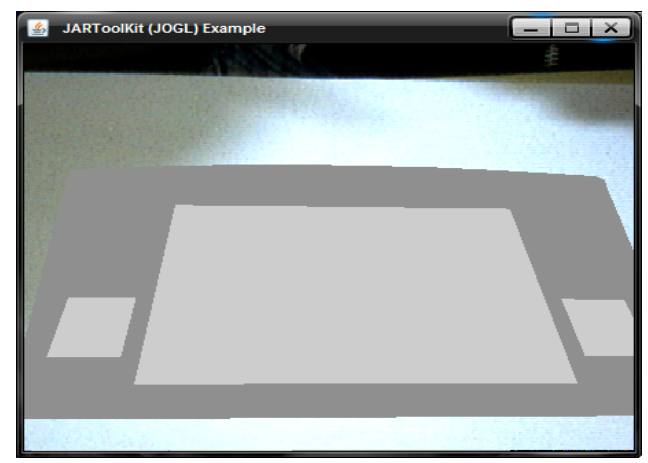

(b)

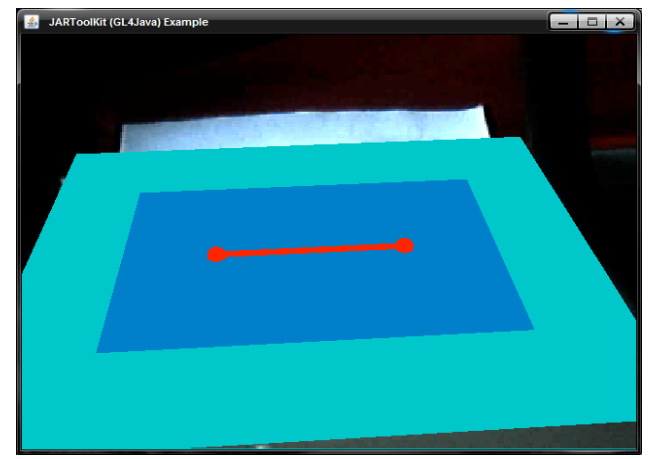

(d)

Figura 6. Caneta e tablet virtuais em OpenGL [Xavier, 2008]

Assim, optou-se por utilizar objetos virtuais modelados em formato OBJ (Wavefront $^{\mathrm{TM}}$ ), como mostram as Figuras 6.a e 6.b, e foram utilizadas duas classes para carregar modelos de objetos virtuais em OBJ desenvolvidas por Pournaras (2006).

Esses objetos também foram modelados em OpenGL a fim de obter uma comparação quanto ao desempenho visual. Posteriormente, apenas os objetos em OpenGL (Figura 6.c e 6.d) foram utilizados pois os outros demandavam mais tempo de carga e visualização.

A Figura 6 mostra, (tanto em cima na 6.a, quanto em baixo na 6.c), o participante segurando a haste (no caso uma caneta sem carga) com o marcador afixado e o software gerando uma caneta virtual (em azul com ponta amarela em 6.c). Deve-se notar que, na haste 
existem 3 marcadores idênticos dispostos de forma que em qualquer posição, mesmo rotacionando a haste, um dos marcadores estará de frente para a webcam. Na Figura 6, à direita (6.b e 6.d), tem-se os tablets virtuais (sobrepondo-se aos respectivos marcadores, que por serem menores, acabam por não aparecer).

A cena é tratada pelo jARToolKit e então apresentada como mostra a Figura 7.a. Deve ser observado na Figura 7.a que na ponta da caneta virtual tem uma pequena esfera amarela e que, quando este coincidir com os pontos vermelhos extremos da reta da tarefa virtual (a uma distância de raio pré-definida e parametrizada), inicia-se efetivamente o teste e o participante deve então tentar seguir a reta até o outro ponto extremo.

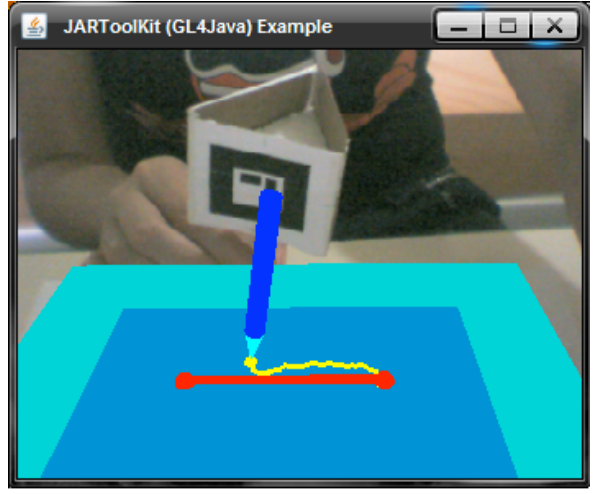

(a)

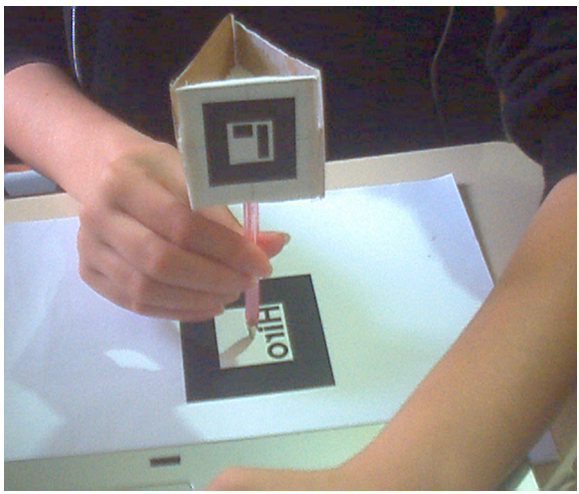

(b)

Figura 7. Setup para utilização da versão em RA

A curva virtual criada pelo participante é desenhada em amarelo sobre o tablet virtual. A Figura 7.b mostra como a câmera "vê" a cena do teste (no caso, no momento da calibração que é feita uma vez por teste/marcador, como será detalhado adiante).

\section{Questões Tecnológicas}

\subsection{Limitações Tecnológicas}

O jARToolKit, possui algumas limitações tecnológicas que podem prejudicar o desempenho de um sistema de RA com marcadores. São elas:

- Alcance da câmera: quanto menor e mais rebuscado for o marcador, mais perto e pior monitorado pela câmera ele será;

- Orientação do marcador em relação à câmera: o marcador é melhor monitorado quando o ângulo entre sua normal e a direção de visualização da 
câmera é de 180 graus; quando este ângulo se distancia deste valor, a câmera fica mais susceptível a erros na identificação do marcador;

- Iluminação: as condições de iluminação afetam a detecção dos marcadores de forma que muita luz pode causar brilho e reflexo no papel, prejudicando a detecção do mesmo, por exemplo; por outro lado, pouca iluminação prejudica a distinção entre os elementos do marcador e sua identificação como um todo, e;

- Instabilidade (jittering) dos objetos na cena: que é uma pequena variação de posição e/ou orientação dos objetos virtuais sem acontecer nenhuma movimentação da câmera nem do marcador; ou seja, os objetos "tremem" na imagem.

As possíveis causas destes problemas são: a resolução, a quantidade de quadros por segundo processados pela câmera, o tipo de iluminação utilizada e as configurações (desempenho) do computador utilizado para a aplicação.

Também cogitou-se que carregar objetos virtuais modelados em OBJ poderia gerar desempenho ("tremor") diferente do que objetos criados usando OpenGL. Por isso, alguns testes foram realizados buscando um melhor desempenho da aplicação basicamente trocando um, ou outro, dos modelos dos objetos e observando-se a instabilidade resultante. Devido a difícil mensuração da instabilidade dos objetos virtuais, esta avaliação foi puramente visual na imagem resultante.

Assim, os objetos virtuais modelados em OBJ foram substituídos por objetos em OpenGL pois estes, criados com geometrias primitivas (nativas à API - Application Programming Interface - do OpenGL) e sem nenhuma textura, "tremiam" menos e apresentaram facilidade de ajustes em sua geometria e posição no espaço 3D contribuindo assim, para a precisão do teste do controle motor.

Quanto a qualidade das câmeras, foi realizado um teste funcional (substituição e configuração para uso no sistema) com a câmera LG Webpro ${ }^{\mathrm{TM}}$, de mais baixa qualidade, e com a Philips ToUcam ${ }^{\text {TM }}$ PRO II modelo PCVC 840K/20, de melhor qualidade. A ToUcam possui resolução de $640 \times 480$ pixels e desempenho de até 30 quadros por segundo e a Webpro possui desempenho de, no máximo, 15 quadros por segundo nesta mesma resolução. Ao realizar o teste, percebeu-se que a instabilidade melhorou muito, sumindo quase que por completo, ao utilizar a câmera ToUcam. Entretanto, o custo para aquisição de uma câmera com tais configurações é mais elevado, apesar de ainda acessível.

Também foi realizada uma simulação de uso para avaliar o aspecto do quanto a iluminação influencia no sistema e verificou-se que a instabilidade dos objetos virtuais aumenta ao utilizar lâmpadas fluorescentes tubulares comparado ao uso de lâmpadas incandescentes. Isso é causado pelo fato de que a lâmpada fluorescente tubular, na verdade, "pisca" a $60 \mathrm{~Hz}$ (frequiência múltipla à de captura da webcam) e atrapalha a visualização do marcador pela câmera. Já a iluminação natural pode prejudicar a realização da tarefa, pois ela 
é instável, variando a quantidade de iluminação ao longo do dia devido a presença de nuvens e reflexos, por exemplo.

\subsection{Problemas de Oclusão do Marcador}

Este problema ocorre quando algum objeto real, sombra ou reflexo de luz sobrepõe um marcador na cena (seja parcial ou total, da caneta ou do tablet) ou quando o marcador sai do alcance da câmera, fazendo com que o sistema não encontre a imagem do marcador nas imagens capturadas pela câmera e, conseqüentemente, não carregue o objeto virtual sobre ele. A oclusão de marcadores é um problema existente tanto no ARToolKit quanto no jARToolKit.Nas versões com RA, o tablet virtual é carregado sobre o marcador e a tarefa deve ser executada com a caneta real sobre a superfície, geralmente cobrindo parcialmente o marcador do tablet e causando a oclusão do mesmo.

Para resolver isso foi necessário encontrar uma forma de "congelar" a matriz interna ao software que identifica o posicionamento do marcador do tablet tornando-o imóvel durante toda a realização da tarefa. Este congelamento foi alcançado através de uma variável booleana que é inicializada no sistema pelo acionamento de uma tecla específica no momento em que o fisioterapeuta define a posição correta (e fixa) para o tablet.

Esta variável controla quando o marcador do tablet deve ser procurado e processado nas imagens capturadas pela câmera (quando a variável está resetada isso leva a recalcular continuamente a matriz de posicionamento do tablet) ou quando a matriz do marcador a ser usada é aquela previamente "congelada" [Xavier, 2008]. Com o posicionamento "congelado" não se tem mais preocupação com oclusão e pode-se até remover o respectivo marcador do tablet que o modelo virtual deste continuará na cena.

\subsection{Calibração do Sistema}

$\mathrm{Na}$ modelagem dos objetos virtuais teve-se a preocupação de que estes fossem semelhantes aos objetos reais e, por isso, o tablet virtual foi modelado nas mesmas dimensões do tablet real. Já a caneta virtual não pôde ser modelada usando este mesmo princípio pois, durante a implementação percebeu-se que os movimentos da caneta são freqüentes, assim como as mudanças de orientação, provocando alterações quanto a tamanho e posição da caneta virtual, dependendo da distância e orientação em relação à câmera [Xavier, 2008]. 


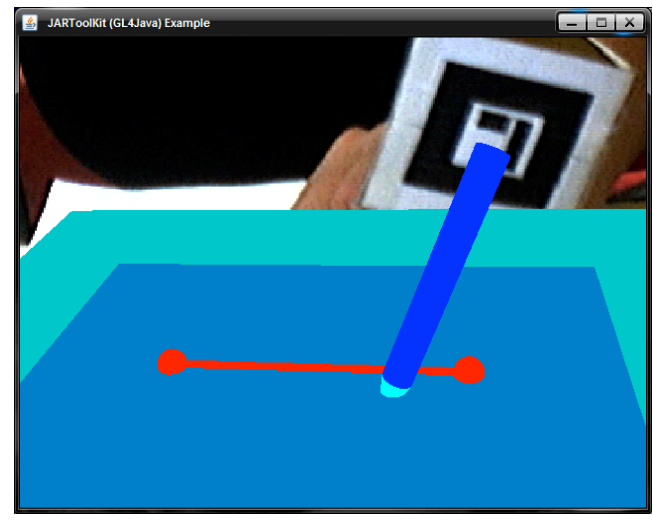

Figura 8. Incoerência entre tablet e caneta virtuais [Xavier, 2008]

Então, a ponta da caneta era parcialmente sobreposta (encoberta) pelo tablet durante alguns momentos (como mostra a Figura 8) mesmo isso não ocorrendo no real (a caneta não perfura o tablet). A solução para este problema foi realizar uma calibração da posição relativa dos objetos virtuais, que consistiu em encontrar a matriz de transformação entre o marcador da caneta e o marcador do tablet.

O Procedimento de calibração foi definido como [Xavier, 2007]:

1. Posiciona-se a webcam em frente ao participante, na altura dos olhos, inclinada verticalmente na direção do marcador do tablet. A câmera deve estar bem em frente ao participante, assim como os marcadores devem estar em uma superfície na frente do participante como mostra a Figura 4.b;

2. Verifica-se se, com esta posição, a câmera captura as imagens do marcador da caneta quando o artefato estiver na posição vertical e com a ponta tocando a superfície. Caso este marcador não esteja visível pela câmera por completo, ajusta-se a webcam de modo que seja possível capturar os marcadores do tablet e da caneta na posição de calibração como mostrada na Figura 7.b;

3. Quando o marcador do tablet estiver posicionado corretamente e apenas este marcador esteja sendo capturado pela câmera, aciona-se uma tecla programada para salvar a matriz de posicionamento deste marcador;

4. Em seguida, posiciona-se a ponta da caneta real sobre o centro do marcador do tablet, garantindo que o marcador não se mova e que o marcador da caneta esteja sendo capturado pela câmera. Uma (outra) tecla específica foi programada para salvar a matriz do marcador da caneta.

A matriz de calibração é calculada multiplicando-se a matriz inversa da matriz do marcador da caneta pela matriz do marcador do tablet. Esta matriz de calibração será multiplicada pela matriz do marcador da caneta a cada quadro do vídeo, obtendo assim, a 
posição e orientação da ponta da caneta virtual em relação ao tablet virtual de forma corrigida/calibrada. A partir desta posição, um cone e um cilindro são modelados dando a forma de uma caneta.

\subsection{Avaliação Geral}

Apesar de que as métricas principais não precisavam ser validadas (pois já haviam sido validadas, como mencionado antes) e as novas métricas aparecem em outros trabalhos (e efetivamente são derivadas das primeiras), o MOSKA tem como diferencial o uso das tecnologias de Banco de Dados para armazenar as informações dos diversos testes (característica não observada nos trabalhos relacionados) e, principalmente, a possibilidade de uso da RA como forma de interação para a captura dos dados.

Observou-se que o nível de "tremor" que persistiu no software com o uso de webcams comuns, foi considerado ainda alto demais para o nível de precisão requerido. Substituir a câmera, usar um computador mais rápido e produzir iluminação controlada levaria a um sistema de custo maior que a própria versão tablet/mouse o que não se justificaria pois sendo assim, a versão RA teria apenas como extra (em relação a versão tablet/mouse) a possibilidade de usar tarefas 3D. Estas tarefas, por sua vez, ainda carecem de maiores estudos quanto a sua real utilidade e validade terapêutica (portanto, é um benefício potencial, não um benefício real).

Mas, o aspecto considerado mais determinante para a não adoção da versão RA baseada em marcadores, como mostrado aqui, está na questão da coordenação visual-motora. Em testes preliminares com os profissionais da área da saúde constatou-se um problema conceitual do setup da tecnologia da RA com marcadores que torna a utilização das implementações feitas aqui questionáveis sob o ponto de vista pragmático do cotidiano de uma clínica: o problema observado foi o desconforto visual causado pela sobreposição dos objetos virtuais sobre a própria mão do participante causando dificuldade na coordenação visualmotora que, para a aplicação em questão, é crítica (Luo et. al., 2005) e pode afetar sobremaneira a confiança nos dados auferidos.

Note-se que tanto com o mouse, com o tablet quanto com a RA, todas estas interações dispõem de uma área para a ação do desenho que deve ser coordenada com uma outra área onde o desenho aparece. Nas duas primeiras somente o próprio desenho é visualizado (ocupando toda a tela) ao passo que na versão RA, o que se observa na tela é a cena como um todo (vista pela webcam, contendo os objetos reais e os objetos virtuais). Então, isso acaba chamando a atenção do participante para a cena do seu processo de desenho mas nesta, ele não consegue se enxergar. Isso tudo o deixa confuso. Essa interação poderia ser aprendida pelo participante com um pouco de prática mas, como se almeja um ambiente intuitivo, de fácil e rápido uso, esta confusão inicial resulta em um limitante do uso da RA na análise do CM fino. 


\section{Conclusão}

Neste artigo foi apresentada a avaliação de técnicas de interação para a análise do controle motor objetivando melhorar a realização do teste para o participante com formas de interação mais intuitivas, interessantes e motivantes. Utilizou-se a Realidade Aumentada (RA) como técnica de interação no auxílio do diagnóstico do controle motor. Foram implementadas três técnicas:

- Na versão "tablet/mouse" foram realizadas melhorias na interface em relação ao ACM [Andrade et al 2003], e incluídas algumas funcionalidades com Banco de Dados e métricas extras;

- Na versão "RA 2D” foi utilizada a RA para capturar dados do controle motor utilizando as mesmas métricas da versão tablet/mouse, e;

- Na versão "RA 3D" tem-se como diferencial o fato de permitir uma tarefa e suas respectivas métricas sendo realizadas no espaço euclidiano 3D (não mais limitados no plano do tablet).

As dificuldades encontradas para implementação das versões RA levaram a soluções como a calibração entre os marcadores da caneta e do tablet virtuais, a modelagem dos objetos virtuais em OpenGL e o "congelamento" do tablet virtual, dentre outras.

A realização do teste do controle motor nas versões com RA foi prejudicada devido às limitações tecnológicas da biblioteca utilizada (jARToolKit), como:

- Sensibilidade à variação de iluminação;

- Freqüente oclusão do marcador por algum objeto, e;

- Restrições entre o ângulo entre o marcador e a câmera.

Além disso, pôde-se perceber que a qualidade da câmera influencia sobremaneira na qualidade de visualização dos objetos virtuais, sendo que uma câmera de baixo custo, com pouca resolução e baixa amostragem de quadros por segundo, aumenta a instabilidade dos objetos virtuais e prejudica a realização do teste de controle motor.

Com as limitações tecnológicas do jARToolKit (comprometendo a robustez da aplicação), com a necessidade de equipamentos melhores (comprometendo o objetivo do baixo custo), com a oclusão da mão do participante (comprometendo a naturalidade no uso), com a necessidade de validar as tarefas em 3D (deixando dúvidas quanto a utilidade) e receios quanto a influência da perda da relação visual-motora (comprometendo a validade), perdeu-se a precisão da tarefa e a intuitividade nas versões RA, se comparadas à versão tablet/mouse.

A princípio, tinha-se a hipótese de que a RA com marcadores e dispositivos de baixo custo trouxesse uma forma mais intuitiva de realizar o teste para o participante. Entretanto, o insucesso na prova desta hipótese não significa que a RA não seja apropriada para este tipo de 
aplicação/interação, e outras formas de RA, que não utilizem marcadores, por exemplo, devem ser experimentadas para confirmar esta hipótese.

Um maior sucesso com a RA na análise do controle motor pode ser obtido ao utilizar uma tecnologia mais avançada e dispositivos de melhor qualidade. Trabalhos futuros nesta direção incluem usar a RA com a capacidade de perceber a relação de proximidade do objeto real e virtual e suas oclusões parciais mas estas se configuram como uma ampla área de investigação da RA que carecem ainda de soluções eficientes e flexíveis.

\section{Referências}

Andrade, A., Haupenthal, A. (2001) "Instrumento de Pesquisa em Aprendizagem Motora: Verificação da Validade e Fidedignidade. Caderno de Resumos da IV Jornada de Pesquisa da UDESC. Florianópolis, p. 90.

Andrade, A., Jardim, L. J. L., Silveira, C. I., Borges Jr., N. G. (2003) “Software de avaliação da aprendizagem e controle motor: aplicação para habilidades motoras finas discretas e fechadas de membros superiores", Em: X Congresso Brasileiro de Biomecânica, Ouro Preto, Anais: Imprensa Universitária UFMG, Vol. II, p. 188-196.

Bueno, J. M. (1998) "Psicomotricidade: Teoria e Prática, Estimulação, Educação e Reeducação Psicomotora com Atividades Aquáticas”. São Paulo: Lovise.

Eder, C. F., Popovic, M. B., Popovic D. B., Stefanovic A., Schwirtlich L., Jovic S. (2005) "The drawing test: assessment of coordination abilities and correlation with clinical measurement of spasticity", In: Archives physical medicine rehabilitation, Vol. 86, número 2, p. 289-295.

Gonçalves, V. P. (2008) "Software de Aprendizagem e Controle Motor para Avaliação de Indivíduos Hemiparéticos: Validade e Confiabilidade". Dissertação de Mestrado. Programa de Ciências do Movimento Humano. UDESC - Universidade do Estado de Santa Catarina. 141 pgs.

Heidemann, E. V. (2008). "Desenvolvimento de Funcionalidades do Software MOSKA". Relatório de Estágio. Curso de Tecnologia em Análise e Desenvolvimento de Sistemas. UDESC - Universidade do Estado de Santa Catarina. 47 pgs.

Hitlab (2008). "Biblioteca ARToolKit". Disponível em:

<http://www.hitl.washington.edu/artoolkit/>, acessado em 12 jun. 2008.

Jardim, L. J. L. (2008). “Concepção De Um Sistema Para Avaliação Da Aprendizagem E Controle Motor: Aplicação Para Habilidades Motoras Finas Discretas E Fechadas De 
Membros Superiores". Trabalho de Conclusão de Curso. Bacharelado em Educação Física. Universidade do Estado de Santa Catarina. 2008. 37 pgs.

Luo, X., Kline, T., Fischer, H. C., Stubblefield, K. A. (2005) "Integration of Augmented Reality and Assistive Devices for Post-Stroke Hand Opening Rehabilitation", Proceedings of the 27th International Conference of the IEEE Engineering and Medicine and Biology Society, Shangai, China, Vol 7, p. 6855-6858.

Pournaras, E. (2006) "Development of a 3D Multiplayer Racing Game", University of Pireaus, Departament of Technology Education and Digital Systems. Disponível em < http://today.java.net/pub/au/489>, acessado em 06 set. 2008.

Sanches, S. O. (2007) "Controle Motor, Dor e Depressão em Mulheres com Síndrome de Fibromialgia", Dissertação (Mestrado em Ciências do Movimento Humano), Universidade do Estado de Santa Catarina, 131 pgs. Florianópolis.

Sherman, W. R., Craig, A. (2003) "Understanding Virtual Reality - Interface, Application and Design", Morgan Kaufmann Publishers.

Sourceforge.net (2008) “jARToolKit”. Disponível em:

<http://sourceforge.net/projects/jartoolkit/>, acessado em 04 ago. 2008.

Subramanian, S; Knaut, L. A., Beaudoin; C., McFadyen, B.J., Feldman A. G. and Levin M. F. (2007) "Virtual Reality Environments for Post-Stroke Arm Rehabilitation", Journal of NeuroEngineering and Rehabilitation, 4:20, junho. Doi: 10.1186/1743-0003-4-20.

Sveistrup, H. (2004) "Motor Rehabilitation using Virtual Reality", Journal of NeuroEngineering and Rehabilitation. 1:10, Dezembro. doi: 10.1186/1743-0003-1-10.

Viau, A., Feldman, A. G., McFadyen, B. J., Levin M. F. (2004) "Reaching in reality and virtual reality: a comparison of kinematics in healthy subjects and in adults with hemiparesis", Journal of NeuroEngineering and Rehabilitation, 1:11, dezembro. doi: 10.1186/1743-0003-1-11.

Xavier, D. C. (2008) “Técnicas de Interação para Análise do Controle Motor", Trabalho de Conclusão de Curso (Bacharelado em Ciência da Computação), Centro de Ciências Tecnológicas, Universidade do Estado de Santa Catarina. SC-Brasil, 119 pgs. 